\title{
Thrombotic Microangiopathy: A Rare Breast Cancer-associated Complication Treated Successfully With Doxorubicin and Cyclophosphamide
}

\author{
SAMUEL JALALI ${ }^{1}$, DAKOTA JENNEMAN ${ }^{2}$, ANKITA TANDON $^{2}$ and HUNG KHONG ${ }^{3}$ \\ ${ }^{1}$ Department of Internal and Hospital Medicine, Moffitt Cancer Center, Tampa, FL, U.S.A.; \\ ${ }^{2}$ Department of Internal Medicine, University of South Florida Morsani College of Medicine, Tampa, FL, U.S.A.; \\ ${ }^{3}$ Department of Medical Oncology, Moffitt Cancer Center, Tampa, FL, U.S.A.
}

\begin{abstract}
Background: Thrombotic microangiopathy (TMA) is a clinical syndrome consisting of hemolytic anemia, thrombocytopenia, and presence of schistocytes on peripheral blood smear secondary to disorders of systemic microvascular thrombosis. Malignancy-associated TMA is a rare entity and shares clinical features with that of HUS and TTP usually seen in patients with metastatic cancer, tumor cell infiltration of the bone marrow and/or response to cancer-directed therapy. Case Report: We present a rare case of TMA secondary to breast cancer without evidence of bone marrow infiltration responsive to doxorubicin and cyclophosphamide treatment, after failed plasmapheresis with prednisone and later, eculizumab. Conclusion: Despite being a rare manifestation of metastatic carcinoma, early identification and treatment are essential to improving survival.
\end{abstract}

Thrombotic microangiopathy (TMA) is a clinical syndrome consisting of hemolytic anemia, thrombocytopenia, and presence of schistocytes on peripheral blood smear secondary to disorders of systemic microvascular thrombosis. The most common causes of TMA include thrombotic thrombocytopenic purpura (TTP), hemolytic uremic syndrome (HUS), and atypical HUS (also known as complement-mediated TMA). TTP is caused by a deficiency in ADAMTS13 (von Willebrand factor cleaving protease), HUS is secondary to Shiga toxin-producing bacteria, and atypical HUS is mediated

This article is freely accessible online.

Correspondence to: Samuel Jalali, MD, Department of Internal and Hospital Medicine, Moffitt Cancer Center, 12902 USF Magnolia Drive, Tampa, FL 33612, USA. Tel: +1 8886633488, email: Samuel.Jalali@moffitt.org

Key Words: Thrombotic microangiopathy, cancer, metastatic breast cancer, microangiopathic hemolytic anemia. by complement activation. These disease processes all result in intravascular thrombi formation, shearing of red blood cells, and endothelial damage. Other etiologies associated with TMA include autoimmune disease, infection, medications, and malignancy (1-3).

Malignancy-associated TMA is a rare entity and shares clinical features with that of HUS and TTP. However, it is usually seen in older patients with metastatic cancer with significantly higher elevations in lactate dehydrogenase (LDH) (4). Tumor cell infiltration of the bone marrow and/or response to cancer-directed therapy have been shown to be key distinguishing factors from other TMA syndromes (4-7). Regierer et al. studied 8 patients who had breast cancerassociated TMA, all patients had bone marrow infiltration of breast cancer, no patient showed ADAMTS13 levels consistent with classic TTP, and 5 out of 6 patients treated with anthracycline therapy showed partial response (3). We present a rare case of TMA secondary to breast cancer without evidence of bone marrow infiltration responsive to doxorubicin and cyclophosphamide treatment, after failed plasmapheresis with prednisone and later, eculizumab.

\section{Case Report}

A 58-year-old female was admitted to our Institution for a 3month history of progressively worsening dyspnea on exertion, generalized fatigue, and skin pallor. Additionally, the patient endorsed lower back pain and cough which preceded symptoms necessitating presentation. She denied fevers, chills, confusion, acute vision changes, chest pain, palpitations, abdominal pain, preceding diarrheal illness, or lower extremity edema. Laboratory evaluation at an outside hospital, three weeks prior to presentation at our institution, included a hemoglobin of $6.9 \mathrm{~g} / \mathrm{dl}$ with normal serum iron, total iron binding capacity (TIBC), ferritin, iron saturation, vitamin B12 and folic acid levels. She was transfused two units of packed red blood cells (pRBC) and discharged. She 
has a past medical history of estrogen receptor-positive, progesterone receptor-negative, and human epidermal growth factor receptor 2 (HER-2 neu)-negative colloid breast adenocarcinoma. She was first diagnosed in 2012, while in Ukraine, and underwent right lumpectomy followed by adjuvant cyclophosphamide, methotrexate, fluorouracil and radiation therapy. Directly after, she was continued on tamoxifen for 2 years, which was discontinued for unknown reasons. In 2016, she was found to have recurrent osseous metastatic disease (confirmed on right sacral biopsy) involving the hip, spine, and skull. She subsequently underwent radiation therapy to the spine and right femur, followed by letrozole and palbociclib. After disease progression, she was switched to exemestane and everolimus, but this regimen was discontinued three weeks prior to presentation for disease progression, with plans to start capecitabine at a later date. On physical examinationn, patient was afebrile with SpO2 99\% on ambient air. Patient was somnolent but easily arousable with icteric sclera and sublingual jaundice. There was no hepatosplenomegaly, rash, purpura or erythema noted. Laboratory evaluation showed a hemoglobin $6.2 \mathrm{~g} / \mathrm{dl}$ hematocrit of $19.5 \%$, MCV of $95 \mathrm{fl}$, platelets $54 \mathrm{k} / \mathrm{ul}$, elevated reticulocyte percent and absolute reticulocyte number of $9.82 \%$ and 0.2013 , respectively, with lactate dehydrogenase (LDH) 1089 U/1, haptoglobin <10 $\mathrm{mg} / \mathrm{dl}$, indirect hyperbilirubinemia (total bilirubin $1.4 \mathrm{mg} / \mathrm{dl}$, indirect bilirubin $1.1 \mathrm{mg} / \mathrm{dl}$ ), and low fibrinogen of 191 $\mathrm{mg} / \mathrm{dl}$. Blood urea nitrogen (BUN) and creatinine were within normal limits. Coagulation studies revealed an elevated prothrombin time (PT) of 13.2 seconds, normal activated partial thromboplastin time (aPTT), and normal international normalized ratio (INR). Direct antiglobulin test (DAT), complement levels and flow cytometry for paroxysmal nocturnal hemoglobinuria were unremarkable. ADAMTS13 activity was slightly low at 59\%; this was ordered on admission but did not result until hospital day 5 . Peripheral blood smear showed presence of polychromasia and schistocytes. Plasmapheresis and prednisone (1 $\mathrm{mg} / \mathrm{kg}$ /day) were started for 4 days for suspected TTP, but this was discontinued as the patient still required $\mathrm{pRBC}$ transfusions, for goal hemoglobin greater than $7 \mathrm{mg} / \mathrm{dl}$, every 1 to 2 days. Bone marrow biopsy on hospital day 12 showed a hypercellular marrow with marked erythroid hyperplasia with no morphologic evidence of metastatic carcinoma or myelodysplastic syndrome. Fluorescence in situ hybridization (FISH) panel was negative. On hospital days 13 and 20, eculizumab, a humanized monoclonal antibody directed against complement protein C5, was administered for suspected atypical HUS. However, she still required frequent $\mathrm{pRBC}$ transfusions. Due to suspicion for breast cancer-associated TMA, doxorubicin and cyclophosphamide (AC) with filgrastim support was administered on hospital day 24. By hospital day 28, transfusion requirements decreased after AC and filgrastim administration. On discharge, hemoglobin stabilized at $7.8 \mathrm{~g} / \mathrm{dl}$ with platelets at $52 \mathrm{k} / \mathrm{ul}$. At her follow-up appointment 15 days after hospitalization, patient's hemoglobin improved to $9.4 \mathrm{mg} / \mathrm{dl}$, platelets to $88 \mathrm{k} / \mathrm{ul}$, total bilirubin at $0.8 \mathrm{mg} / \mathrm{dl}$ and she was noted to have significant reduction in transfusion requirements and increased quality of life. Patient was continued on liposomal doxorubicin after discharge without recurrence of breast cancer-associated TMA.

\section{Discussion}

Cancer-associated TMA is a syndrome consisting of Coombs-negative microangiopathic hemolytic anemia (MAHA) with associated thrombocytopenia and evidence of schistocytes on peripheral blood smear. Adenocarcinoma (particularly mucin-producing) was the most common histologic subtype associated with MAHA, with gastric being the most common, followed by breast, prostate and lung. In addition, a proportion of these tumors produce mucin, including benign neoplasms $(1,8-10)$. It should be noted that our patient presented with a lesser common malignancyassociated TMA, and had a colloid (i.e. mucin-producing) adenocarcinoma of the breast, which has been reported sparsely in the literature (10-11). Further characteristics of cancer-associated TMA are listed in Table I.

There are no diagnostic criteria available when evaluating for cancer-related TMA. It can be difficult to distinguish from other TMA syndromes with similar features, such as TTP/HUS. TTP consists of the classic pentad of fever, MAHA, thrombocytopenia, renal failure and neurologic deficits, whereas HUS presents as MAHA, thrombocytopenia and renal failure (7). Some key characteristics that distinguish cancer-related TMA from TTP is evidence of active or recurrent malignancy, older age at presentation (usually in the mid-50s versus early-40s with TTP), ADAMTS13 activity is generally closer to $50 \%$ (rather than $<5-10 \%$ ), failure to respond to plasmapheresis, and presenting complaints usually include bone/back pain and dyspnea $(2,7)$. Our patient presented with all of these findings. In addition, our patient had normal complement levels with failure to respond to eculizumab and no preceding bloody diarrheal illness, making atypical HUS and HUS unlikely, respectively.

A more prominent feature of cancer-related TMA is cancer involvement of the bone marrow. Although all patients in the Regierer et al. study had bone marrow infiltration, in a literature review of 51 patients with breast cancer-related TMA, $71 \%$ of cases had evidence of bone marrow involvement (10). Similar cases published after this publication either did not perform a bone marrow biopsy or were unable to yield a sufficient sample for analysis. This indicates that breast cancer-associated TMA is possible in the absence of bone marrow infiltration, but this is an 
Table I. Compilation of literature related to breast cancer-associated TMA.

\begin{tabular}{|c|c|c|}
\hline Author/Year & Study & Findings \\
\hline Regierer et al. 2011 (3) & Case Series & $\begin{array}{l}\text { - } 8 \text { patients diagnosed with breast cancer-associated TMA } \\
\text { - All patients had bone marrow infiltration of breast cancer } \\
\text { - No patient had severely low ADAMTS13 levels consistent with classic TTP } \\
\text { - } 5 / 6 \text { patients treated with anthracycline therapy showed partial response }\end{array}$ \\
\hline Lechner and Obermeier 2012 (8) & Case Series & $\begin{array}{l}\text { - Out of } 168 \text { cases, adenocarcinoma was the most common histologic subtype } \\
\text { associated with MAHA, with gastric being the most common, followed by } \\
\text { breast, prostate and lung } \\
\text { - } 14 \text { were associated with lymphoma } \\
\text { - Over } 90 \% \text { of cases were associated with metastatic cancer } \\
\text { - Higher survival rates seen in those who received antineoplastic therapy }\end{array}$ \\
\hline Takabatake and Oishi 2016 (9) & $\begin{array}{l}\text { Case Report/ } \\
\text { Literature Review }\end{array}$ & $\begin{array}{l}\text { Metastatic breast cancer patient who was previously on chemotherapy with } \\
\text { normalization of tumor markers and near resolution of metastases } \\
\text { - Presented with MAHA with recurrent increase in tumor markers, } \\
\text { with osseous but no visceral metastases } \\
\text { - Anemia initially improved with paclitaxel, followed by eribulin } \\
\text { - Of } 51 \text { patients with breast cancer related TMA, } \\
71 \% \text { of cases had bone marrow involvement }\end{array}$ \\
\hline Lee et al. 2019 (11) & Case Report & $\begin{array}{l}\text { - Metastatic breast cancer patient presented with MAHA, normal ADAMTS13 } \\
\text { levels, LDH levels }>2,500 \mathrm{U} / 1 \text { and peripheral smear with schistocytes } \\
\text { - Persistent hyperbilirubinemia and minimal improvement } \\
\text { in platelet counts with plasma exchange } \\
\text { - Normalization of bilirubin and resolution of schistocytes after } \\
3 \text { doses of doxorubicin/paclitaxel }\end{array}$ \\
\hline
\end{tabular}

TMA: Thrombotic microangiopathy; TTP: thrombotic thrombocytopenic purpura; MAHA: microangiopathic hemolytic anemia; LDH: lactate dehydrogenase.

uncommon scenario. Our patient's lack of bone marrow involvement of her tumor highlights the rarity of our case.

When evaluating cancer patients with TMA, chemotherapy complications should also be considered. Reports of TMA have been commonly associated with gemcitabine and mitomycin, as well as oxaliplatin. When suspected, the treatment is to stop all chemotherapeutic agents $(2,7,12)$. Our patient had been taken off all chemotherapy 3 weeks prior to presentation with persistence of symptoms, lowering suspicion for chemotherapy-related TMA.

Although the pathophysiology is better understood for most other TMA syndromes (i.e. TTP/HUS), it is not clearly understood in the context of metastatic cancers. It has been theorized that bone marrow infiltration from tumor cells and abnormal angiogenesis invade the bone marrow's vasculature, causing endothelial cell injury, releasing large von Willebrand factor (vWF) multimers. This coupled with development of autoantibodies against ADAMTS13, result in intravascular microthrombi that directly shear red blood cells. Alternatively, the proposed endothelial damage and associated vWF multimer aggregation could be induced from tumor emboli or within small vessels of cancerous tissue (78 ). One case of breast cancer-associated TMA proposed carcinocythemia as a possible mechanism after finding circulating cancer cells collided with red blood cells on peripheral blood smear (13). Mucin also has been hypothesized to play a pathophysiologic role (1). Since our patient did not have tumor cell infiltration of her bone marrow, we suspect that the mucin produced by their cancer, and endothelial damage from cancer cells played a significant physiologic role in developing TMA.

The mainstay of treatment for cancer-related TMA is treating the underlying malignancy. In breast cancer, improvements in cell counts and rates of survival have been seen with anthracycline therapy, like our patient $(8,10,14)$. However, some patients deteriorate quickly, precluding patients from receiving appropriate treatment on time. Cancer-related TMA has a high mortality rate (50\%), making early identification and treatment essential to improving survival (11). Therapies, including plasma exchange and eculizumab, have been proposed for the treatment of cancer related TMA, with no proven efficacy. The lack of improvement with plasma exchange is likely due to the lack of a significant decrease in ADAMTS13 activity compared to that of TTP $(7,10,15)$. Cancer-related TMA is not associated with a severe deficiency of ADAMTS13 activity 
(14). However, ADAMTS13 lab results usually take days to return (like our patient), and thus, it is reasonable to initiate empiric plasma exchange if peripheral blood smear reveals schistocytes. A partial improvement with plasma exchange in a patient with signet cell breast cancer-associated TMA has been reported (11).

\section{Conclusion}

Given the presence of a TMA, in the setting of no bone marrow infiltration of carcinoma and borderline-low ADAMTS13, with positive response to doxorubicin and cyclophosphamide, it can be assumed that peripheral circulating tumor cells and/or cancerous tissue may have deleterious effects on erythrocytes, platelets, and possibly, their precursors. There are no diagnostic criteria for cancerassociated TMA, but diagnosis should be suspected in a patient with the following: active malignancy (especially metastatic adenocarcinoma), DAT-negative hemolytic anemia, thrombocytopenia, presence of schistocytes with other causes excluded, and ADAMTS13 activity not consistent with TTP (less than 5 to 10\%). Despite being a rare manifestation of metastatic carcinoma, early identification and treatment are essential to improving survival.

\section{Conflicts of Interest}

The Authors declare that there are no conflicts of interest in relation to this case report.

\section{Authors' Contributions}

S Jalali: Literature research, proofreading, editing and writing of the article. D Jenneman: Literature research, editing, proofreading and writing of the article. A Tandon: Literature research and writing of the article. H Khong: Editing and proofreading of the article.

\section{References}

1 Kristian P, Zdravko P, Rogosic Veljko M, Kovacic V, Marijana $\mathrm{M}$, Damir A, Roko $\mathrm{M}$ and Zeljko $\mathrm{N}$ : Thrombotic microangiopathy secondary to a mucinous cystic neoplasm of the pancreas. Ann Clin Case Rep 4: 1707, 2019.

2 Morton JM and George JN: microangiopathic hemolytic anemia and thrombocytopenia in patients with cancer. J Oncol Pract 12(6): 523-530, 2016. PMID: 27288467. DOI: 10.1200/JOP. 2016.012096

3 Regierer AC, Kuehnhardt D, Schulz CO, Flath B, Jehn CF, Scholz CW, Possinger K and Eucker J: Breast cancer-associated thrombotic microangiopathy. Breast Care (Basel) 6(6): 441-445, 2011. PMID: 22419897. DOI: 10.1159/000335201

4 Francis KK, Kalyanam N, Terrell DR, Vesely SK and George $\mathrm{JN}$ : Disseminated malignancy misdiagnosed as thrombotic thrombocytopenic purpura: A report of 10 patients and a systematic review of published cases. Oncologist 12(1): 11-19, 2007. PMID: 17227897. DOI: 10.1634/theoncologist.12-1-11
5 George JN: How I treat patients with thrombotic thrombocytopenic purpura: 2010. Blood 116(20): 4060-4069, 2010. PMID: 20686117. DOI: 10.1182/blood-2010-07-271445

6 George JN: Systemic malignancies as a cause of unexpected microangiopathic hemolytic anemia and thrombocytopenia. Oncology (Williston Park) 25(10): 908-914, 2011. PMID: 22010388.

7 Govind Babu K and Bhat GR: Cancer-associated thrombotic microangiopathy. Ecancermedicalscience 10: 649, 2016. PMID: 27433282. DOI: $10.3332 /$ ecancer.2016.649

8 Lechner K and Obermeier HL: Cancer-related microangiopathic hemolytic anemia: Clinical and laboratory features in 168 reported cases. Medicine (Baltimore) 91(4): 195-205, 2012. PMID: 22732949. DOI: 10.1097/MD.0b013e3182603598

9 Lohrmann HP, Adam W, Heymer B and Kubanek B: Microangiopathic hemolytic anemia in metastatic carcinoma. Report of eight cases. Ann Intern Med 79(3): 368-375, 1973. PMID: 4748253. DOI: 10.7326/0003-4819-79-3-368

10 Takabatake D and Oishi K: Microangiopathic hemolytic anemia associated with metastatic breast cancer: case report and literature review. Springerplus 5(1): 684, 2016. PMID: 27350919. DOI: $10.1186 / \mathrm{s} 40064-016-2312-4$

11 Lee EH, Otoukesh S, Abdi Pour A and Nagaraj G: Hemolytic anemia of malignancy: a case study involving signet ring cell metastatic breast cancer with severe microangiopathic hemolytic anemia. Case Rep Oncol 12(1): 104-108, 2019. PMID: 31043946. DOI: $10.1159 / 000494753$

12 Daviet F, Rouby F, Poullin P, Moussi-Francès J, Sallée M, Burtey S, Mancini J, Duffaud F, Sabatier R, Pourroy B, Grandvuillemin A, Grange S, Frémeaux-Bacchi V, Coppo P, Micallef J and Jourde-Chiche N: Thrombotic microangiopathy associated with gemcitabine use: Presentation and outcome in a national French retrospective cohort. Br J Clin Pharmacol 85(2): 403-412, 2019. PMID: 30394581. DOI: 10.1111/bcp.13808

13 Robier C, Neubauer M, Beham-Schmid C and Sill H: Thrombotic microangiopathy and disseminated intravascular coagulation associated with carcinocythemia in a patient with breast cancer. J Clin Oncol 29(34): e825-e826, 2011. PMID: 22025152. DOI: 10.1200/JCO.2011.36.7433

14 Fontana S, Gerritsen HE, Kremer Hovinga J, Furlan M and Lämmle B: Microangiopathic haemolytic anaemia in metastasizing malignant tumours is not associated with a severe deficiency of the von Willebrand factor-cleaving protease. Br J Haematol 113(1): 100-102, 2001. PMID: 11328288. DOI: 10.1046/j.1365-2141.2001.02704.x

15 Werner TL, Agarwal N, Carney HM and Rodgers GM: Management of cancer-associated thrombotic microangiopathy: What is the right approach? Am J Hematol 82(4): 295-298, 2007. PMID: 17034030. DOI: 10.1002/ajh.20783 